\section{Perfil dos pesquisadores da área de odontologia no Conselho Nacional de Desenvolvimento Científico e Tecnológico (CNPq)}

\section{Profile of dentistry researchers of the Brazilian National Research Council (CNPq)}

\section{Resumo}

Pesquisadores brasileiros têm demonstrado aumento na produção científica associada à publicação de trabalhos na literatura corrente. $\mathrm{O}$ objetivo desse estudo foi compreender o perfil de pesquisadores brasileiros voltados para a odontologia com bolsas de produtividade concedidas pelo Conselho Nacional de Pesquisa (CNPq). Para revelar o perfil, 132 Currículos Lattes de pesquisadores, durante 2003 a 2005, foram cuidadosamente analisados, e variáveis como gênero, classificação no CNPq (1A a 1D, 2), instituição de origem, tempo de doutorado, artigos publicados com o respectivo Qualis, livros e capítulos de livros, orientações de mestrado e doutorado e áreas de atuação foram catalogados. O perfil geral foi formado por homens (64,39\%), classificação classe $2(42,42 \%)$, concentrados em instituições do Estado de São Paulo (85,6\%), com 10 a 15 anos de doutorado (32.57\%), publicando em periódicos Qualis A Internacional e Qualis B Nacional. A produção de livros ou capítulos de livros, orientações de mestrados ou doutorados foram heterogêneas. A visão geral revelou uma produção consistente associada a grupos de pesquisadores consolidados.

Palavras-Chave: Produção científica. Pesquisadores. Pesquisa odontológica.
Raika Augusta Cavalcante

Daniella Reis Barbosa ${ }^{2}$

Paulo Rogério Ferreti Bonan²

Maria Betânia de Oliveira Pires ${ }^{2}$

Hercílio Martelli-Júnior ${ }^{2}$

${ }^{1}$ Faculdades Unidas do Norte de Minas Gerais, Montes Claros, Minas Gerais, Brasil.

${ }^{2}$ Centro de Ciências Biológicas e da Saúde, Universidade Estadual de Montes Claros, Uimontes, Montes Claros, Minas Gerais, Brasil.

Correspondência: Hercílio Martelli Júnior, Rua Iracy de Oliveira Novaes, 220 - Apt 207-A - Cândida Câmara, Montes Claros, Minas Gerais, CEP 39400-000, E-mail: hmjunior2000@yahoo.com
Aprovado pelo Comitê de Ética em Pesquisa da Universidade Estadual de Montes Claros, Resolução №. 021 - CEPEX/2007 


\section{Abstract}

Brazilian researchers have been showing increasing scientific production associated with the publication of studies in the literature. The aim of this study was to understand the profile of Brazilian researchers in dentistry with productivity grants from the Brazilian National Research Council (CNPq). One hundred and thirty two Lattes curricula, from 2003 to 2005 , were carefully analyzed by gender, $\mathrm{CNPq}$ classification (1A to 1D, 2), institution of origin, year of $\mathrm{PhD}$ degree, number of papers published with their respective Qualis classification, number of books and book chapters, number of MSc and PhD supervisions, and area of work. The general profile was comprised by males (64.39\%), level 2 of CNPq classification (42.42\%), concentrated in institutions in the State of São Paulo (85.6\%), with 10 to 15 years of $\mathrm{PhD}$ degree (32.57 \%), publishing on Qualis A international journals and Qualis B national journals. The production of books, book chapters and MSc and PhD supervisions was heterogeneous. The general overview revealed consistent production associated with consolidated groups of researchers.

Keywords: Scientific production. Research personnel. Dentistry Research.

\section{Introdução}

De um modo geral, o aumento da produção científica odontológica brasileira pode ser verificado pelo crescimento do número de artigos científicos publicados em periódicos odontológicos por pesquisadores afiliados a universidades brasileiras, no MEDLINE, que é o maior acervo internacional de revistas científicas sobre saúde ${ }^{1}$. Esse aumento observado não é apenas numérico absoluto, mas também uma elevação percentual em relação a outros países, como confirmado por dados do ISI e do SciELO².

O desenvolvimento da pesquisa apresenta importante papel para a geração de novos conhecimentos, novas tecnologias e para o desenvolvimento do espírito crítico e reflexivo na formação acadêmica do profissional $^{3}$. Um dos responsáveis por esse aumento da produção científica nacional é o sistema de pós-graduação, que, por intermédio da Coordenação de Aperfeiçoamento de Pessoal de Nível Superior (CAPES), prioriza o número de artigos publicados para conceituar os programas nacionais ${ }^{4}$.

Com a expansão da produção científica e formação de recursos humanos, através da pós-graduação, há expressivo aumento na demanda por recursos de financiamento para projetos de investigação e bolsas de produtividade em pesquisa, bem como solicitação de bolsas de pós-doutorado e bolsas "sanduíche" junto à CAPES. Com o crescimento constante observado na área de saúde coletiva, a proporção de bolsistas de produtividade tende a representar parcela cada vez menor no conjunto de pesquisadores, e a pressão por bolsas de produtividade tende a aumentar levando à adoção de critérios cada vez mais restritivos $^{5}$. Baseado no aumento da produção científica odontológica brasileira, este estudo tem como objetivo estabelecer o perfil dos pesquisadores em odontologia, com bolsas de produtividade científica, no CNPq, no triênio de 2003 a 2005. 


\section{Metodologia}

O presente estudo é de caráter transversal e descritivo. Para sua realização foi utilizada a relação dos bolsistas de produtividade do CNPq, na área de odontologia, com bolsas ativas no triênio de 2003 a 2005. A partir da identificação dos bolsistas, foram consultados os Currículos Lattes de todos os pesquisadores para cada uma das categorias vigentes no CNPq, 2, 1A, 1B, 1C, 1D e sênior. Foram excluídos dessa análise pesquisadores que se encontravam com bolsas suspensas, como nos casos de realização de pós-doutorado no exterior.

A partir dos currículos Lattes, acessados na plataforma Lattes do CNPq, foi construído banco de dados com informações relativas à distribuição dos pesquisadores por categoria $(2,1 \mathrm{~A}, 1 \mathrm{~B}, 1 \mathrm{C}, 1 \mathrm{D}$ e sênior), distribuição geográfica e institucional, tempo de conclusão do curso de doutorado, produção científica (artigos científicos, livros e capítulos de livros) nacional e internacional, formação de recursos humanos (orientação de iniciação científica, mestrado e doutorado) e principais áreas de atuação na odontologia.

Todos os parâmetros analisados corresponderam ao período de 2003 a 2005 e para a classificação dos artigos científicos foi adotada a padronização da Capes, pelo sistema Qualis. As últimas consultas aos currículos Lattes foram realizadas entre os meses de abril e agosto de 2006, período suficiente para as atualizações dos pesquisadores.

\section{Resultados e discussão}

$\mathrm{O}$ universo de pesquisadores do $\mathrm{CNPq}$ do presente estudo foi de 132 bolsistas ativos, sendo $85(64,39 \%)$ do gênero masculino e $47(35,61 \%)$ do gênero feminino, distribuídos nas seis possíveis classes de bolsistas do CNPq. Observa-se que não houve pesquisadores bolsistas na classe sênior (Tabela 1). A maior concentração de bolsistas está na classe $2(42,42 \%)$, ou seja, aqueles pesquisadores, com no mínimo 2 anos de obtenção do título de doutor. Mesmo quando se analisa o gênero em relação à categoria do bolsista, verifica-se que em todas as cinco classes encontradas de pesquisadores, houve predomínio do gênero masculino. Nas categorias 1A, 1B e 1C as diferenças foram bastante evidentes (Tabela 1). Com relação à idade dos bolsistas, não há menção no currículo Lattes, não possibilitando a aferição dessa variável.

Tabela 1 - Distribuição dos bolsistas da área de odontologia, segundo gênero e a categorização do CNPq.

Table 1 - Distribution of dentistry researchers with grants, according to gender and $\mathrm{CNPq}$ category.

\begin{tabular}{lccc}
\hline Categoria & $\begin{array}{c}\text { Masculino } \\
(\mathrm{n})\end{array}$ & $\begin{array}{c}\text { Feminino } \\
(\mathrm{n})\end{array}$ & $\begin{array}{c}\text { Total }(\mathrm{n}) \\
(\%)\end{array}$ \\
\hline 2 & 30 & 26 & $56(42,4)$ \\
1D & 13 & 9 & $22(16,7)$ \\
$1 \mathrm{C}$ & 14 & 6 & $20(15,1)$ \\
1B & 16 & 3 & $19(14,4)$ \\
1A & 12 & 3 & $15(11,4)$ \\
Sênior & 0 & 0 & 0 \\
Total & 85 & 47 & $132(100)$ \\
\hline
\end{tabular}

Os pesquisadores bolsistas estão distribuídos por 11 diferentes Estados da federação, sendo que apenas na região Sudeste estão presentes 113 bolsistas (85,6\%), sendo 101 do Estado de São Paulo. Em seguida, observam-se 10 bolsistas nos Estados do Sul, respectivamente, em Santa Catarina, Rio Grande do Sul e Paraná (Tabela 2). Esses dados podem ser atribuídos à maior concentração de cursos de graduação e pós-graduação em odontologia nesses Estados da federação. Particularmente no Estado de São Paulo estão concentrados os cursos de maiores conceitos na pós-graduação odontológica brasileira ${ }^{6}$. Quando se observa a Tabela 3, em relação à Tabela 2, verifica-se que dos 101 bolsistas do Estado de São Paulo, 100 estão distribuídos nas três Instituições Públicas Estaduais. Exceto a UNICAMP, que possui apenas um curso de odontologia, localizado na cidade de Piracicaba (FOP- 
UNICAMP), a USP e a UNESP possuem em conjunto 6 cursos de odontologia, sendo 3 da USP (Bauru, São Paulo e Ribeirão Preto) e 3 da UNESP (Araçatuba, Araraquara e São José dos Campos). As três Instituições Estaduais Paulistas também concentram o maior número de programas de pós-graduação no país ${ }^{6}$. Dos 132 pesquisadores bolsistas, $126(95,45 \%)$ estão vinculados a Instituições de Educação Superior Públicas, sendo 103 bolsistas de Instituições Públicas Estaduais e 23 de Instituições Públicas Federais (Tabela 3).

Com relação ao tempo médio de obtenção do título de Doutor, as maiores concentrações de pesquisadores bolsistas situam-se, respectivamente, entre 10 a 15 anos (32,57\%) e 5 a 10 anos (25\%). Por outro lado, a menor presença de bolsistas se dá abaixo dos 5 anos de obtenção do doutoramento $(5,3 \%)$ (Tabela 4$)$. A Tabela 5 refere-se à produção científica nacional e internacional, em periódicos e livros. A citação dos artigos científicos teve como referência o Sistema Qualis da Capes ${ }^{6}$. Observa-se nessa Tabela a média trienal de artigos científicos nacionais e internacionais gerados por cada classe de pesquisador. Verifica-se, de modo geral, amplo predomínio de artigos Qualis B (52,05\%),

Tabela 2 - Distribuição geográfica (Estados da federação) dos bolsistas da área de odontologia, no CNPq.

Table 2 - Geographic distribution (federation states) of dentistry researchers with grants from CNPq.

\begin{tabular}{lcc}
\hline Estado da Federação & $\mathrm{n}$ & $\%$ \\
\hline São Paulo & 101 & 76,5 \\
Minas Gerais & 6 & 4,5 \\
Rio de Janeiro & 6 & 4,5 \\
Santa Catarina & 5 & 3,8 \\
Rio Grande do Sul & 4 & 3,0 \\
Pernambuco & 3 & 2,3 \\
Rio Grande do Norte & 2 & 1,5 \\
Paraíba & 2 & 1,5 \\
Bahia & 1 & 0,7 \\
Paraná & 1 & 0,7 \\
Pará & 1 & 0,7 \\
Total & 132 & 100 \\
\hline
\end{tabular}

Tabela 3 - Distribuição dos pesquisadores bolsistas do CNPq, segundo a Instituição de atuação.

Table 3 - Distribution of dentistry researchers with grants from $C N P q$, according to Institution.

\begin{tabular}{lcc}
\hline Instituição de Educação & $\mathrm{n}$ & $\%$ \\
Superior (IES) & & \\
\hline USP & 46 & 34,8 \\
FOP-UNICAMP & 31 & 23,5 \\
UNESP & 23 & 17,4 \\
UFMG & 5 & 3,8 \\
UFSC & 4 & 3,0 \\
UFRN & 2 & 1,5 \\
UFF & 2 & 1,5 \\
UFRJ & 2 & 1,5 \\
UFPB & 2 & 1,5 \\
UPE & 2 & 1,5 \\
UFPe & 2 & 1,5 \\
UNESA & 2 & 1,5 \\
PUC-PR & 1 & 0,7 \\
UFPE & 1 & 0,7 \\
PUC-RS & 1 & 0,7 \\
UFU & 1 & 0,7 \\
UFPA & 1 & 0,7 \\
UFBA & 1 & 0,7 \\
UNOESC & 1 & 0,7 \\
UNAERP & 1 & 0,7 \\
UFP & 132 & 0,7 \\
Total & 100 \\
\hline
\end{tabular}

em comparação com o Qualis A (24,07\%) e C $(23,86 \%)$, respectivamente. Quando se analisa por classe de pesquisador, $1 \mathrm{C}$ foi $\mathrm{o}$ que mostrou maior produção científica, em periódicos nacionais e 1A em periódicos internacionais. Com relação aos periódicos nacionais Qualis A e C, houve maior predomínio de autores da classe $1 \mathrm{~A}$. Na publicação em periódicos internacionais, destaca-se o evidente predomínio de artigos científicos do Qualis A (66,56\%), em comparação com os Qualis B e C. Os pesquisadores bolsistas do 1A foram responsáveis pela maior concentração de artigos científicos nos periódicos A e B internacionais. Ainda no indicador produção científica, a Tabela 5 apresenta a publicação de livros ou capítulos de livros pelos bolsistas pesquisadores. Verifica-se nítida pre- 
Tabela 4 - Distribuição dos pesquisadores bolsistas do CNPq, segundo o tempo de conclusão do doutorado.

Table 4 - Distribution of dentistry researchers with grants from $C N P q$, according to time from doctorate completion.

\begin{tabular}{lcccccc}
\hline Tempo (anos) & 2 & $1 \mathrm{D}$ & $1 \mathrm{C}$ & $1 \mathrm{~B}$ & $1 \mathrm{~A}$ & Total \\
\hline Até 5 & 7 & 0 & 0 & 0 & 0 & 7 \\
$5-10$ & 19 & 7 & 4 & 2 & 1 & 33 \\
$10-15$ & 19 & 6 & 7 & 7 & 4 & 43 \\
$15-20$ & 8 & 5 & 5 & 4 & 1 & 23 \\
$20-30$ & 3 & 1 & 3 & 4 & 2 & 13 \\
Acima de 30 & 0 & 3 & 1 & 2 & 7 & 13 \\
Total & 56 & 22 & 20 & 19 & 15 & 132 \\
\hline
\end{tabular}

Tabela 5 - Produção científica média dos pesquisadores bolsistas do CNPq, no triênio, em periódicos nacionais*, internacionais*, livros e capítulos de livros ${ }^{* *}$.

Table 5 - Average of scientific production of dentistry researchers with grants from CNPq, in the past three years, in the domestic and international literature, books and chapters*.

\begin{tabular}{lcccccc}
\hline Periódico (Qualis) & 2 & $1 \mathrm{D}$ & $1 \mathrm{C}$ & $1 \mathrm{~B}$ & $1 \mathrm{~A}$ & Média \\
\hline Nacional A & 1,7 & 2,3 & 2,7 & 2,1 & 2,9 & 2,3 \\
Nacional B & 3,8 & 5,3 & 8,0 & 4,4 & 3,9 & 5,1 \\
Nacional C & 2,3 & 2,4 & 2,6 & 1,2 & 3 & 2,3 \\
Internacional A & 7,5 & 4,2 & 9,1 & 11 & 12,8 & 8,9 \\
Internacional B & 1,4 & 1 & 1,3 & 1,3 & 2 & 1,4 \\
Internacional C & 2,4 & 2,9 & 4,6 & 3,5 & 3,4 & 3,4 \\
Capítulo & 1,3 & 1,1 & 2,4 & 2,1 & 2,7 & 1,9 \\
Livro & 0,1 & 0,1 & 0,2 & 0,6 & 0,5 & 0,3 \\
\hline
\end{tabular}

*Média de artigos científicos nacionais e internacionais por classe de pesquisador, através do total parcial dividido pelo número de bolsistas em cada categoria.

* Average of domestic and international scientific papers according to researcher class: partial total divided by number of researchers with grants in each category.

**Média de livros e capítulos de livros por classe de pesquisador, através do total parcial dividido pelo número de bolsistas em cada categoria.

** Average of books and chapters according to researcher class:partial total divided by the number of researchers with grants in each category

dominância na publicação de capítulos de livros (85,71\%), em comparação com livros $(14,29 \%)$, no triênio analisado. A classe $1 \mathrm{~A}$ foi a que publicou mais capítulos de livros, enquanto a $1 \mathrm{~B}$ foi a que publicou mais livros no triênio avaliado.

Outro importante parâmetro na atividade científica dos pesquisadores bolsistas pode ser ilustrado na Tabela 6. Essa tabela refere-se à formação de recursos humanos por parte dos pesquisadores bolsistas, ou seja, a orientação e formação de alunos em nível de iniciação científica, mestrado e doutorado. Verifica-se que, no triênio analisado, houve prevalência na formação de mestres $(42,76 \%)$, seguida de alunos de iniciação científica $(30,32 \%)$ e doutores (26,91\%). Entre as classes de pesquisadores, a $1 \mathrm{C}$ foi a que apresentou no conjunto a maior parcela de orientações, seguida pela classe 1B. Em relação à orientação de iniciação científica, a maior concentração se deu na classe 2 e mestrado na classe 1A. Esses valores apresentados foram corrigidos pelo número de bolsistas em cada classe de pesquisador.

Com relação à área de atuação na odontologia dos pesquisadores bolsistas, o currículo Lattes permite a inserção de mais de uma opção para cada pesquisador. Assim, a Tabela 7 ilustra as principais especialidades da odontologia obtidas através dos cur- 
Tabela 6 - Formação de recursos humanos (orientação de iniciação científica, mestrado e doutorado) dos pesquisadores bolsistas do CNPq, no triênio*.

Table $\mathbf{6}$ - Average of human resources trained (orientation of scientific initiation and stricto sensu postgraduation) by researchers with grants from $C N P q$, in the past three years.

\begin{tabular}{lcccccc}
\hline Orientação & 2 & $1 \mathrm{D}$ & $1 \mathrm{C}$ & $1 \mathrm{~B}$ & $1 \mathrm{~A}$ & Média \\
\hline Iniciação Científica & 3,4 & 2,4 & 2,7 & 2,5 & 1,6 & 2,5 \\
Mestrado & 2,6 & 3,1 & 4,5 & 3 & 4,6 & 3,6 \\
Doutorado & 1,9 & 1,7 & 2,3 & 3,1 & 2,2 & 2,2 \\
Média Total & 2,6 & 2,4 & 3,2 & 2,9 & 2,8 & 2,8 \\
\hline
\end{tabular}

rículos dos pesquisadores. Destaca-se a área de biomateriais ou materiais odontológicos, que se insere em praticamente todas as especialidades da odontologia, seguida da área de clínica odontológica, que também é bastante extensa, por contemplar diversas especialidades, tais como endodontia, periodontia, prótese e pediatria. A área de patologia enquadra, entre outras, as citações de patologia bucal, estomatologia e semiologia. Um estudo que enfatizou a pesquisa odontológica no Estado de Minas Gerais, avaliando a apresentação de projetos de pesquisa em editais universais da Fundação de Amparo à Pesquisa do Estado de Minas Gerais (FAPEMIG), demonstrou que as áreas de Odontologia Restauradora e Patologia Bucal foram as mais expressivas em número de propostas apresentadas. Isso estava em concordância com a produção mais freqüente nessas áreas, neste estudo ${ }^{7}$. Em outra análise que contemplou a cruzamento de dados referentes aos trabalhos apresentados na $16^{a}$ Reunião Anual da Sociedade Brasileira de Pesquisa Odontológica (SBPqO), com publicações posteriores, evidenciou-se que as áreas de maior publicação completa foram referentes a pesquisas in vitro com Materiais Dentários e Dentística corroborando os nossos achados $^{8}$. Além disso, verifica-se uma expressiva concentração de programas de pós-graduação na CAPES, tendendo a enfatizar os domínios profissionalizantes do conhecimento odontológico ${ }^{9}$. Desse modo, algumas áreas na odontologia carecem de programas de pós-graduação, tais como saúde coletiva, odontologia legal e deontologia ${ }^{7}$.
Tabela 7 - Distribuição dos pesquisadores bolsistas do CNPq, segundo as áreas de atuação, citadas no currículo Lattes.

Table 7 - Distribution of researchers with grants from CNPq, according to area, quoted in Lattes curriculum.

\begin{tabular}{lcc}
\hline Área de Atuação na Odontologia & $\mathrm{n}$ & $\%$ \\
\hline Materiais Odontológicos & 36 & 16 \\
Clínica Odontológica & 33 & 15 \\
Patologia (Diagnóstico Bucal) & 23 & 10 \\
Endodontia & 14 & 6 \\
Periodontia & 14 & 6 \\
Saúde Coletiva & 10 & 5 \\
Odontopediatria & 10 & 5 \\
\hline
\end{tabular}

Embora não esteja representado em tabelas, foram analisados os periódicos nacionais e internacionais mais utilizados para publicação pelos bolsistas. Entre os periódicos nacionais, os cinco mais encontrados foram, respectivamente, Journal of Applied Oral Science, Brazilian Dental Journal, Brazilian Oral Research, Revista de Pós-Graduação da FO-USP e Ciência Odontológica Brasileira. Os três primeiros dessa relação possuem também indexação internacional e são publicados em inglês. Os dois primeiros, Journal of Applied Oral Science e Brazilian Dental Journal, são editados, respectivamente, pela USP Bauru e Ribeirão Preto. O terceiro, Brazilian Oral Research, está vinculado à Sociedade Brasileira de Pesquisa Odontológica. No caso dos periódicos internacionais, os cinco mais encontrados nas publicações dos bolsistas foram, respectivamente, Journal of Periodontology, Operative Dentistry, Dental Materials, 
International Endodontic Journal e Oral Surgery, Oral Medicine, Oral Pathology, Oral Radiology and Endodontology. O primeiro dos periódicos internacionais é da área de periodontia, sendo órgão oficial da Academia Americana de Periodontia. O segundo e terceiro, Operative Dentistry e Dental Materials, estão mais centrados na área de biomateriais, prótese e dentística. O quarto periódico dessa série internacional, International Endodontic Journal, é da área de endodontia, e o último, Oral Surgery, Oral Medicine, Oral Pathology, Oral Radiology and Endodontology, está voltado predominantemente à área de patologia bucal, radiologia odontológica e cirurgia, contemplando também a área de endodontia. É importante ressaltarmos a tendência para a publicação em periódicos escritos em língua inglesa. Em dois trabalhos realizados na Argentina que verificaram a produção odontológica associada a um evento similar à SBPqO e posterior publicação, em intervalos temporais distintos, evidenciaram a tendência dos autores a privilegiar periódicos internacionais $^{10-11}$. Mesmo quando a publicação de escolha é nacional, as indexações internacionais, que muitas vezes são sinônimo de publicação em inglês, são um fator atrativo para os autores ${ }^{8}$. É importante ressaltar a presença de alguns problemas metodológicos referentes às informações coletadas, como já foi destacado ${ }^{5}$. Assim, como as informações foram obtidas exclusivamente do currículo Lattes dos pesquisadores bolsistas, pode haver diferenças no preenchimento das informações pelos pesquisadores. Entretanto, como o currículo Lattes é um dos elementos decisivos no julgamento e avaliação de bolsas, e na captação de recursos financeiros em editais de pesquisa, considerou-se que seria a melhor fonte para caracterização do perfil dos bolsistas ${ }^{5}$. Outro aspecto a ser pontuado na Metodologia do estudo diz respeito à superestimação relativa da produção científica, visto que, em alguns casos, os mesmos produtos gerados possuem mais de um autor, e dois ou mais auto- res podem ser bolsistas ${ }^{5}$.

De modo geral, a produção científica odontológica brasileira tem aumentado ${ }^{1}$. O aumento do número de propostas enviadas por odontólogos para os editais de pesquisa da FAPEMIG, por exemplo, constitui evidência da aculturação progressiva dos docentes de ensino superior sobre a necessidade de produzir e publicar ${ }^{7}$. O mesmo fenômeno incremental foi observado na produção científica odontológica argentina, ainda que de forma relativamente polarizada, como ocorre no Brasil ${ }^{10}$. Embora o aumento da produção científica brasileira seja claramente mensurável, em termos de publicação, utilizando critérios quantitativos e qualitativos, o Brasil ainda se encontra atrás dos países escandinavos, dos Estados Unidos, do Reino Unido e do Japão ${ }^{12}$. É importante que, junto a esse salto positivo na quantidade, haja uma busca pela qualidade. Para que isso ocorra, é necessário que pesquisadores identifiquem problemas relevantes com base em teorias sólidas e interessantes, formulem hipóteses específicas, e que dados sejam coletados e analisados para testá-las ${ }^{1}$. Além disso, o incremento na produção científica é permeado necessariamente pelo aprimoramento da pós-graduação no exercício da confecção e escrita do trabalho científico ${ }^{4}$. Acrescendo, o papel do incentivo financeiro, como aquele associado às bolsas, também tem impacto positivo na produção publicada dos pesquisadores e no seu envolvimento maior com o desenvolvimento de novos trabalhos científicos ${ }^{13}$.

Alguns aspectos relevantes passíveis de serem pontuados no presente estudo dizem respeito a:

- maior predomínio nitidamente verificado entre bolsistas do gênero masculino, comparado ao feminino;

- distribuição bastante evidente da maioria dos bolsistas no Estado de São Paulo, particularmente nas três instituições estaduais, USP, UNICAMP e UNESP;

- prevalência dos pesquisadores vinculados às Instituições Públicas Estaduais e Federais, onde se está concentrada a mai- 
oria dos programas de pós-graduação; busca pela literatura científica internacional para publicação de seus estudos, porém com produção também destacada na literatura científica nacional, sobretudo naqueles periódicos com indexação internacional; e

- destaque para pesquisas na área de biomateriais, com aumento importante da área da saúde coletiva, conforme já foi observado $^{1}$.
Faz-se importante enfatizar que os dados aqui apresentados referentes aos pesquisadores bolsistas da área de odontologia fossem comparados com os de outras áreas do conhecimento, a partir de metodologias similares, permitindo inclusive conhecer melhor as áreas e talvez definir estratégias de demandas induzidas de pesquisa.

\section{Referências}

1. Nadanovsky P. O aumento da produção científica odontológica brasileira na saúde pública. Cad Saúde Pública 2006; 22: 886.

2. Souza PR. O crescimento da produção científica. Folha de São Paulo 2002; nov 06. p. A3.

3. Péret ACM, Lima MRL. A pesquisa e a formação do professor de odontologia nas políticas internacionais e nacionais de educação. Revista ABENO 2005; 3: 65-9.

4. Volpato GL, Freitas EG. Desafios na publicação científica. Pesqui Odontol Bras 2003; 17: 49-56.

5. Barata RB, Goldbaum M. Perfil dos pesquisadores com bolsa de produtividade em pesquisa do CNPq da área de saúde coletiva. Cad Saúde Pública 2003; 19:1863-76.

6. Coordenação de Aperfeiçoamento de Pessoal de Nível Superior (Capes). http://www.capes.gov.br [acessado em 22 de outubro de 2006].

7. Martelli-Júnior H, Vieira Júnior JR, Domingos MA, Barbosa DR, Brito-Júnior M, Bonan PR. Pesquisa Odontológica no Estado de Minas Gerais-Brasil: Análise retrospectiva de 1986 a 2006. Arquivos em Odontologia 2007; 43: 23-9.
8. Leles CR, Rocha SS, Simões PA, Compagnoni MA. Taxa de publicação, na forma de artigos completos, de resumos apresentados em evento científico de pesquisa odontológica. Revista de Odontologia da UNESP 2006; 35 : 141-7.

9. Carvalho ACP, Kriger L. Educação Odontológica. São Paulo: Editora Artes Médicas Ltda; 2006.

10. Collet AM, Jara-Tracchia L, Palacios SB, Itoiz ME. Dental research productivity in Argentina (1993 to 2003). Acta Odontol Latinoam 2006; 19: 81-4

11. Collet AM, Piloni MJ, Keszler A. Scientific presentations and publications on odontological research in Argentina. Acta Odontol Latinoam 1997; 10: 63-9.

12. Gil-Montoya JA, Navarrete-Cortes J, Pulgar R, Santa S, Moya-Anegón F. World dental research production: an ISI database approach (1999-2003). Eur J Oral Sci 2006; 114: 102-8.

13. Iacopino AM, Pryor ME, Taft TB, Lynch DP. The effect of NIDCR R25 grant support on the curriculum and culture of a research non-intensive dental school. J Dent Res 2007; 86: 581-5.

Recebido em: 16/03/07 Versão final reapresentada em: 09/12/07 Aprovado em: 19/12/07 